\title{
GENEALOGY FOR SUPERCRITICAL BRANCHING PROCESSES
}

\author{
ANDREAS NORDVALL LAGERÅS *** AND \\ ANDERS MARTIN-LÖF,* Stockholm University
}

\begin{abstract}
We study the genealogy of so-called immortal branching processes, i.e. branching processes where each individual upon death is replaced by at least one new individual, and conclude that their marginal distributions are compound geometric. The result also implies that the limiting distributions of properly scaled supercritical branching processes are compound geometric. We exemplify our results with an expression for the marginal distribution for a class of branching processes that have recently appeared in the theory of coalescent processes and continuous stable random trees. The limiting distribution can be expressed in terms of the Fox $H$-function, and in special cases by the Meijer $G$-function.
\end{abstract}

Keywords: Supercritical branching process; compound geometric distribution; Fox $H$-function; Meijer $G$-function

2000 Mathematics Subject Classification: Primary 60J80

Secondary 60E07; 60F05; 33C60

\section{Introduction}

A branching process is a Markov process in continuous time. Heuristically it can be considered as the number of particles in a population where the particles behave independently of each other, live for exponentially distributed periods of time, and at death give birth to new particles according to some distribution on the nonnegative integers. Usually the population is assumed to start with one particle. The name 'branching process' is appropriate since we can describe the evolution of the population by drawing a family tree in which the lifetime of each particle corresponds to a particular branch.

We shall mainly consider branching processes where each particle gives birth to at least two new particles. We call such processes immortal branching processes since they cannot become extinct. Our main result is that the number of individuals in an immortal branching processes has a compound geometric distribution. We obtain a corollary which states that the limiting distributions of properly scaled supercritical branching processes are compound geometric.

We will start by recalling some well-known results about branching processes and compound distributions in Sections 2 and 3, respectively. In Section 4, we give a proof and a new interpretation of the old result that immortal branching processes are infinitely divisible. With a detailed study in Section 5 of the so-called Yule process, we can in Section 6 give our main result, Theorem 2, a similar proof and interpretation as in Section 4.

Received 31 March 2006; revision received 12 June 2006.

* Postal address: Department of Mathematics, Stockholm University, Stockholm, SE-10691, Sweden.

** Email address: andreas@math.su.se 
It is generally hard to find explicit expressions for marginal distributions for branching processes. In Section 7, we exemplify our results with a branching process that has recently appeared in the theory of coalescent processes and continuous stable random trees.

\section{Branching processes}

We can describe the dynamics of a branching process $Z=\left\{Z_{t}\right\}_{t \geq 0}$, where the number of new particles at each birth has the distribution $\left\{p_{k}\right\}_{k \geq 0}$, as follows (see [1]). The process starts with $Z_{0}=1$. If the process is in state $i$ at any time, then it remains there for an amount of time which is exponentially distributed with parameter $i \mu$, where $\mu$ is the intensity of the process, and then jumps into state $j \geq i-1$ with probability $p_{j-i+1}$. It then stays in state $j$ for an exponentially distributed time with parameter $j \mu$, and jumps to state $k \geq j-1$ with probability $p_{k-j+1}$, and so on.

Let $f(s)=\sum_{k=0}^{\infty} p_{k} s^{k}$ be the generating function (GF) of the distribution $\left\{p_{k}\right\}$. The expected number of new particles at each birth is given by $m=f^{\prime}(1)$. A necessary and sufficient condition for the process described above not to explode in finite time almost surely, i.e. $\mathrm{P}\left(Z_{t}<\infty\right)=1$, is that

$$
\int_{1-\varepsilon}^{1} \frac{\mathrm{d} s}{f(s)-s}
$$

diverges for all $\varepsilon, 0<\varepsilon<1$; see [5]. A sufficient condition for this to hold is $m<\infty$.

The Kolmogorov forward equation for the GF $F(s, t)=\mathrm{E}\left[s^{Z_{t}}\right]$ is

$$
\frac{\partial}{\partial t} F(s, t)=\mu(f(s)-s) \frac{\partial}{\partial s} F(s, t) .
$$

From this equation, we see that it is no loss of generality to assume that $p_{1}=0$ since, if $Z$ is a branching process with $p_{1}>0$ and intensity $\mu$, then it is distributed in the same way as the branching process $Z^{*}$ with intensity $\mu^{*}=\mu\left(1-p_{1}\right)$ and GF for the offspring

$$
f^{*}(s)=\frac{f(s)-p_{1} s}{1-p_{1}}=\sum_{k=0}^{\infty} p_{k}^{*} s^{k},
$$

where $p_{1}^{*}=0$ and $p_{k}^{*}=p_{k} /\left(1-p_{1}\right)$ for $k=0,2,3, \ldots$ We henceforth exclude the trivial case $p_{1}=1$ from our analysis. Thus, we can, and will, assume that $p_{1}=0$.

As $t \rightarrow \infty$, the branching process almost surely either dies out, i.e. $Z_{t} \rightarrow 0$, or explodes, i.e. $Z_{t} \rightarrow \infty$. The behaviour we will observe depends only on the expected number of offspring of each particle. If $m \leq 1$ then the process dies out almost surely. If $m>1$ then there is a positive probability, $1-q$, of explosion at infinity and the process is called supercritical. It is easily shown that $q$, the probability of extinction, is the smallest nonnegative root of $q=f(q)$.

Our first result shows that, in the case of a supercritical branching process, we can scale it in time to obtain a nontrivial random variable in the limit.

Proposition 1. With notation as above, let $\lambda=\mu(m-1)$, with $1<m<\infty$. There exists a nonnegative random variable $W$ such that

$$
\mathrm{e}^{-\lambda t} Z_{t} \rightarrow W \text { almost surely }
$$

as $t \rightarrow \infty$. Furthermore, $W \neq 0$ if and only if

$$
\sum_{k=2}^{\infty} p_{k} k \log k<\infty \text {. }
$$


For a proof of Proposition 1, see [1].

We shall, in particular, study branching processes with $p_{0}=0$. We call such processes immortal branching processes, since they are nondecreasing and, thus, $q=0$. Since $\left\{p_{k}\right\}$ is a distribution on $\{2,3, \ldots\}$, we can write its GF as $f(s)=s g(s)=s^{2} k(s)$, where $g(s)$ and $k(s)$ are GFs for distributions on $\mathbb{N}$ and $\mathbb{N}_{0}$ (the nonnegative integers), respectively. The Kolmogorov backward equation for the GF can thus be written as

$$
\begin{aligned}
\frac{\partial}{\partial t} F(s, t) & =\mu(f(F(s, t))-F(s, t)) \\
& =\mu F(s, t)(g(F(s, t))-1) \\
& =\mu F(s, t)(F(s, t) k(F(s, t))-1) .
\end{aligned}
$$

There is a connection between supercritical processes conditioned on exploding and immortal branching processes. Consider the supercritical branching process $Z$. At any given time this process will have individuals of two types, those who will have an infinite line of descent and those who will not. Let $\widetilde{Z}_{t}$ be the number of the former individuals at time $t$. Since the individuals behave independently, conditional on $Z_{t}=n$ we have $\widetilde{Z}_{t} \sim \operatorname{Bin}(n, 1-q)$; furthermore, $\widetilde{Z}=\left\{\widetilde{Z}_{t}\right\}_{t>0}$ is itself an immortal branching process if it is positive (see [1]).

The exact relation between the limiting distribution of Proposition 1 for an exploding branching process $Z$ and the associated immortal process $\widetilde{Z}$ is given by the following result.

Proposition 2. The proportion $\widetilde{Z}_{t} / Z_{t}$ will, conditional on $Z$ exploding, converge almost surely to $1-q$ as $t \rightarrow \infty$, where $q$ is the extinction probability of the supercritical branching process.

For a proof of Proposition 2, see [1, Sections I.12 and III.7]. Because of this result, it is sufficient to study immortal branching processes to understand the limiting behaviour of supercritical branching processes conditioned on exploding.

\section{Compound distributions}

We recall some results about compound distributions. All random variables in this section are assumed to be nonnegative. More details can be found in [8]. A random variable $X$ is compound- $N$ if

$$
X \stackrel{\mathrm{D}}{=} \sum_{i=1}^{N} Y_{i},
$$

where ' $\stackrel{\mathrm{D}}{=}$ ' denotes equality in distribution, $N$ is a random variable with distribution on $\mathbb{N}_{0}$, and $Y_{1}, Y_{2}, \ldots$ are independent and identically distributed (i.i.d.). The empty sum is defined to be zero. Let $g_{N}(s)=\mathrm{E}\left[s^{N}\right]$ be the GF of $N$ and let $L_{Y}(\theta)=\mathrm{E}\left[\mathrm{e}^{-\theta Y_{1}}\right]$ be the Laplace-Stieltjes transform of the distribution of $Y_{1}$. Then the Laplace-Stieltjes transform (of the distribution) of $X$ is

$$
L_{X}(\theta)=\mathrm{E}\left[\mathrm{e}^{-\theta X}\right]=\mathrm{E}\left[\mathrm{E}\left[\mathrm{e}^{-\theta X} \mid N\right]\right]=\mathrm{E}\left[L_{Y}(s)^{N}\right]=g_{N}\left(L_{Y}(\theta)\right) .
$$

If $Y_{1}$ has a distribution on $\mathbb{N}_{0}$, then we can write the GF as $g_{X}(s)=g_{N}\left(g_{Y}(s)\right)$. Some examples of discrete compound distributions are the compound Poisson distribution with GF

$$
F(s)=\exp (v(G(s)-1)), \quad v>0,
$$

and the compound geometric distribution with GF

$$
F(s)=\frac{1-p}{1-p H(s)}, \quad 0<p<1,
$$


where $G(s)$ and $H(s)$ are some GFs. Since

$$
\begin{aligned}
\frac{1-p}{1-p H(s)} & =\exp \left(-\log (1-p)\left(\frac{\log (1-p H(s))}{\log (1-p)}-1\right)\right) \\
& =\exp \left(-\log (1-p)\left(g_{N}(H(s))-1\right)\right),
\end{aligned}
$$

where $g_{N}(s)$ is the GF of the logarithmic distribution, we see that all compound geometric random variables are also compound Poisson. Note that if $X$ has GF $F(s)$ then $\widetilde{F}(s)=$ $(F(s)-F(0)) /(1-F(0))$ is the GF of $X$, given that $X>0$, with $\widetilde{F}(0)=0$. By rescaling $v$ and $p$, we can always choose $G(0)=H(0)=0$ in (6) and (7), respectively.

The probability distribution of the random variable $X$ is said to be infinitely divisible if, for all positive integers $n$, there exist some i.i.d. random variables $X_{1}, \ldots, X_{n}$ such that $X \stackrel{\mathrm{D}}{=} X_{1}+\cdots+X_{n}$. This is equivalent to $L_{X}(\theta)=L_{X_{1}}(\theta)^{n}$, where $L_{X_{1}}(\theta)$ is the LaplaceStieltjes transform of $X_{1}$. The compound Poisson distributions are important in part due to the following proposition, a proof of which can be found in [8].

Proposition 3. All infinitely divisible random variables $X$ with $\mathrm{P}(X=0)>0$ are compound Poisson, and all infinitely divisible distributions can be obtained as the weak limit of compound Poisson distributions. Furthermore, all weak limits of infinitely divisible distributions are infinitely divisible.

For a noninteger-valued random variable $N$, we define $X$, a compound- $N$ variable, as follows. Let $Y$ be infinitely divisible. Thus, $L_{Y}(\theta)^{a}$ is a Laplace-Stieltjes transform for all $a>0$. We define $X$ by its Laplace-Stieltjes transform

$$
L_{X}(\theta)=\mathrm{E}\left[L_{Y}(\theta)^{N}\right]=\mathrm{E}\left[\exp \left(N \log L_{Y}(\theta)\right)\right]=L_{N}\left(-\log L_{Y}(\theta)\right) .
$$

If $N$ is infinitely divisible then so is $X$. The compound exponential distribution has LaplaceStieltjes transform

$$
L_{X}(\theta)=\frac{\lambda}{\lambda-\log L_{Y}(\theta)}=\frac{1}{1-\log L_{Y}(\theta)^{1 / \lambda}},
$$

which we obtain with $N \sim \operatorname{Exp}(\lambda)$. Note that we can always choose $\lambda=1$ by changing $Y$. Compound exponential distributions are infinitely divisible, since the exponential distribution is infinitely divisible. The following result is an analogue of Proposition 3 for compound exponential and compound geometric distributions.

Proposition 4. All compound exponential random variables $X$ with $\mathrm{P}(X=0)>0$ are compound geometric, and all compound exponential distributions can be obtained as the weak limit of compound geometric distributions. Furthermore, all weak limits of compound exponential distributions are compound exponential.

The second and third statements of this proposition can be proved, for example, by using the continuity theorem for Laplace-Stieltjes transforms. The first statement is easily checked. Since

$$
0<\mathrm{P}(X=0)=\lim _{\theta \rightarrow \infty} L_{X}(\theta)=\lim _{\theta \rightarrow \infty} \frac{1}{1-\log L_{Y}(\theta)},
$$

we obtain $\lim _{\theta \rightarrow \infty} L_{Y}(\theta)=\mathrm{P}(Y=0)>0$. Since $Y$ is infinitely divisible, it follows, by Proposition 3, that $Y$ is compound Poisson, so $L_{Y}(\theta)=\exp \left(v\left(L_{V}(\theta)-1\right)\right)$ for some random variable $V$. We obtain

$$
L_{X}(\theta)=\frac{1}{1-\log L_{Y}(\theta)}=\frac{1}{1-v\left(L_{V}(\theta)-1\right)}=\frac{1-v /(1+v)}{1-(v /(1+v)) L_{V}(\theta)} .
$$


We will also need the following result.

Lemma 1. If $X$, given that $X>0$, is compound exponential, then $X$ is compound geometric.

Proof. Let $p=\mathrm{P}(X>0)$. We obtain

$$
\begin{aligned}
L_{X}(\theta) & =(1-p) L_{X \mid X=0}(\theta)+p L_{X \mid X>0}(\theta) \\
& =1-p+\frac{p}{1-\log L_{Y}(\theta)} \\
& =\frac{1-p}{1-p(1 /(1-p)) /\left(1 /(1-p)-\log L_{Y}(\theta)\right)} \\
& =\frac{1-p}{1-p L_{V}(\theta)}
\end{aligned}
$$

where

$$
L_{V}(\theta)=\frac{1 /(1-p)}{1 /(1-p)-\log L_{Y}(\theta)}
$$

clearly is a Laplace-Stieltjes transform of a compound exponential probability distribution.

\section{Infinite divisibility}

Theorem 1. All immortal branching processes are infinitely divisible.

Proof. From (4), we obtain

$$
\frac{\partial}{\partial t} \log \left(\frac{F(s, t)}{s}\right)=\mu(g(F(s, t))-1)
$$

and

$$
\begin{aligned}
\frac{F(s, t)}{s} & =\exp \left(\mu\left(\int_{0}^{t} g(F(s, u)) \mathrm{d} u-t\right)\right) \\
& =\exp \left(\mu t\left(\int_{0}^{t} g(F(s, t-r)) \frac{\mathrm{d} r}{t}-1\right)\right)
\end{aligned}
$$

which is the GF of a compound Poisson distribution since $\int_{0}^{t} g(F(s, t-r)) \mathrm{d} r / t$ is a GF. Since $Z_{t}-1$ is compound Poisson, it is infinitely divisible; thus, $Z_{t}$ is infinitely divisible.

The distribution (8) has an interesting probabilistic interpretation. Imagine that the ancestor has a certain title that he passes on to only one of his offspring when he dies. Each individual who has the title passes it on in the same way when he dies. Up to time $t$, the title will have been passed on a number of times which is Poisson distributed with mean $\mu t$. The distribution of the number of siblings of a title-bearer will have GF $g(s)$, and if a title-bearer was born at time $r$, then each of his siblings will be an ancestor of a number of individuals with GF $F(s, t-r)$ at time $t$. Thus, the total contribution of a birth of a title-bearer at time $r$ to the final size of the branching process at time $t$ will have GF $g(F(s, t-r))$. We finally note that each time of birth of a title-bearer will be uniformly distributed over $(0, t)$, with probability density $1 / t$, if we disregard the order of the times.

Corollary 1. Let $Z$ be a supercritical branching process and $W$ be the limiting random variable of Proposition 1. Assume that (3) holds. Then $W$, given that $W>0$, has an infinitely divisible distribution. 
Proof. Let $\widehat{W}$ have the distribution of $W$, given that $W>0$, and let $\widetilde{Z}$ be the branching process whose individuals are those individuals of $Z$ that have an infinite line of descent. As noted earlier, $\widetilde{Z}$ is an immortal branching process. Since $\widetilde{Z}_{t}$ is infinitely divisible for all $t$, so is $\mathrm{e}^{-\lambda t} \widetilde{Z}_{t}$, which, by Proposition 3, implies that $\widetilde{W}=\lim _{t \rightarrow \infty} \mathrm{e}^{-\lambda t} \widetilde{Z}_{t}$ is also infinitely divisible. Now, $\widehat{W}=\widetilde{W} /(1-q)$, where $q=\mathrm{P}(W=0)$; thus, even $\widehat{W} \stackrel{\mathrm{D}}{=}(W \mid W>0)$ is infinitely divisible.

Remark 1. Earlier proofs of the infinite divisibility of an immortal branching process and its limiting random variable can be found in [3]. Our proof is different, and our interpretation of the result also prepares us for proving further results, such as Theorem 2.

\section{The Yule process}

The simplest example of an immortal branching process is the so-called Yule process, with $p_{2}=1$. The Yule process is one of a few branching processes whose marginal distribution can be found explicitly. One way of finding the distribution is by solving the Kolmogorov backward equation, but a more direct approach provides the joint distribution of the number of individuals in the process at time $t$, and their times of birth. This additional information will be useful later on. Let $0=\tau_{(0)}<\tau_{(1)}<\cdots$ be the times of birth in the Yule process.

Lemma 2. The distribution of $Z_{t}-1$ is geometric with parameter $1-\mathrm{e}^{-\mu t}$, and the distribution of $\left(\tau_{(1)}, \ldots, \tau_{(n)}\right)$ conditional on $Z_{t}-1=n$ is the same as an ordered sample of i.i.d. random variables $\tau_{1}, \ldots, \tau_{n}$ with probability density

$$
h(r)=\frac{\mathrm{P}\left(\tau_{k} \in \mathrm{d} r\right)}{\mathrm{d} r}=\frac{\mu \mathrm{e}^{-\mu(t-r)}}{1-\mathrm{e}^{-\mu t}} .
$$

Proof. Let $\left(t_{1}, \ldots, t_{n+1}\right) \in(0, t)^{n+1}$ and let $\left(t_{(1)}, \ldots, t_{(n+1)}\right)$ be the ordered sample of $\left(t_{1}, \ldots, t_{n+1}\right)$; set $t_{(0)}=0$. Recall that $\tau_{(k)}-\tau_{(k-1)} \sim \operatorname{Exp}(k \mu)$ for $k \geq 1$. We obtain

$$
\begin{aligned}
\mathrm{P}\left(\tau_{(1)}\right. & \left.\in \mathrm{d} t_{(1)}, \ldots, \tau_{(n)} \in \mathrm{d} t_{(n)}, \tau_{(n+1)}>t\right) \\
& =\prod_{k=1}^{n} \mathrm{P}\left(\tau_{(k)} \in \mathrm{d} t_{(k)} \mid \tau_{(k-1)}=t_{(k-1)}\right) \mathrm{P}\left(\tau_{(n+1)}>t \mid \tau_{(n)}=t_{(n)}\right) \\
& =\prod_{k=1}^{n} k \mu \exp \left(-k \mu\left(t_{(k)}-t_{(k-1)}\right)\right) \exp \left(-(n+1) \mu\left(t-t_{(n)}\right)\right) \mathrm{d} t_{(1)} \cdots \mathrm{d} t_{(n)} \\
& =n ! \mu^{n} \exp \left(-\mu\left((n+1) t-\sum_{k=1}^{n} t_{(k)}\right)\right) \mathrm{d} t_{(1)} \cdots \mathrm{d} t_{(n)} \\
& =n ! \mathrm{e}^{-\mu t} \prod_{k=1}^{n} \mu \exp \left(-\mu\left(t-t_{(k)}\right)\right) \mathrm{d} t_{(1)} \cdots \mathrm{d} t_{(n)} \\
& =\mathrm{e}^{-\mu t}\left(1-\mathrm{e}^{-\mu t}\right)^{n} n ! \prod_{k=1}^{n} h\left(t_{(k)}\right) \mathrm{d} t_{(1)} \cdots \mathrm{d} t_{(n)} \\
& =\mathrm{e}^{-\mu t}\left(1-\mathrm{e}^{-\mu t}\right)^{n} \prod_{k=1}^{n} h\left(t_{k}\right) \mathrm{d} t_{1} \cdots \mathrm{d} t_{n} .
\end{aligned}
$$


Since $\mathrm{P}\left(Z_{t}-1=n\right)=\mathrm{P}\left(\tau_{(n)} \leq t<\tau_{(n+1)}\right)$, we see from (10) or (11) that $Z_{t}-1$ has a geometric distribution with parameter $1-\mathrm{e}^{-\mu t}$. Furthermore, we see from (10) that the joint distribution of the times of birth has the density $n ! \prod_{k=1}^{n} h\left(t_{(k)}\right)$; thus, they can be seen as an ordered sample of i.i.d. random variables $\tau_{1}, \ldots, \tau_{n}$ with probability density $h(r)$.

We note that $\tau_{k} \stackrel{\mathrm{D}}{=} t-\varepsilon$ conditional on $\varepsilon<t$ for an $\varepsilon \sim \operatorname{Exp}(\mu)$. This suggests the following algorithm for generating the ages of the individuals in a Yule process at time $t$.

- Draw i.i.d. random variables $\varepsilon_{1}, \varepsilon_{2}, \ldots$ from $\operatorname{Exp}(\mu)$.

- Stop as soon as any $\varepsilon_{n}>t$.

- Set $Z_{t}=n$ and let the ages of the individuals be the realizations of $\varepsilon_{k}$ for $1 \leq k \leq n-1$, with the original ancestor of course having age $t$.

The GF for the distribution of $Z_{t}-1$ is

$$
\frac{F(s, t)}{s}=\frac{\mathrm{e}^{-\mu t}}{1-\left(1-\mathrm{e}^{-\mu t}\right) s},
$$

which we also could have found by solving the Kolmogorov backward equation. More generally, we can also find the distribution when $p_{k+1}=1$ for $k \geq 2$. Here we have

$$
\frac{F(s, t)}{s}=\left(\frac{\mathrm{e}^{-\mu k t}}{1-\left(1-\mathrm{e}^{-\mu k t}\right) s^{k}}\right)^{1 / k},
$$

so $Z_{t}-1$ has the distribution of a multiple of a negative binomial random variable. We can also find the distribution of the limiting variable $W$ of Proposition 1 . We note that $\lim _{t \rightarrow \infty} \mathrm{e}^{-\lambda t} Z_{t}=$ $\lim _{t \rightarrow \infty} \mathrm{e}^{-\lambda t}\left(Z_{t}-1\right)$ and that $\lambda=\mu(m-1)=\mu k$. The Laplace-Stieltjes transform of $W$ is given by

$$
\begin{aligned}
L_{W}(\theta) & =\lim _{t \rightarrow \infty} \mathrm{E}\left[\exp \left(-\theta \mathrm{e}^{-\mu k t}\left(Z_{t}-1\right)\right)\right] \\
& =\lim _{t \rightarrow \infty}\left(\frac{\mathrm{e}^{-\mu k t}}{1-\left(1-\mathrm{e}^{-\mu k t}\right) \exp \left(-k \theta \mathrm{e}^{-\mu k t}\right)}\right)^{1 / k} \\
& =\left(\frac{1}{1+k \theta}\right)^{1 / k},
\end{aligned}
$$

so the distribution is gamma, and, in particular, the distribution is exponential for the limit of the Yule process.

\section{Further distributional properties}

The results of Section 5 are in concordance with Theorem 1 and Corollary 1, but we note that the distributions obtained are not only infinitely divisible but also compound geometric, and compound exponential in the limit. This is in fact true for all immortal branching processes.

Theorem 2. All immortal branching processes have compound geometric distributions.

Proof. We make the ansatz (compare with (12))

$$
\frac{F(s, t)}{s}=\frac{\mathrm{e}^{-\mu t}}{1-\left(1-\mathrm{e}^{-\mu t}\right) H(s, t)},
$$




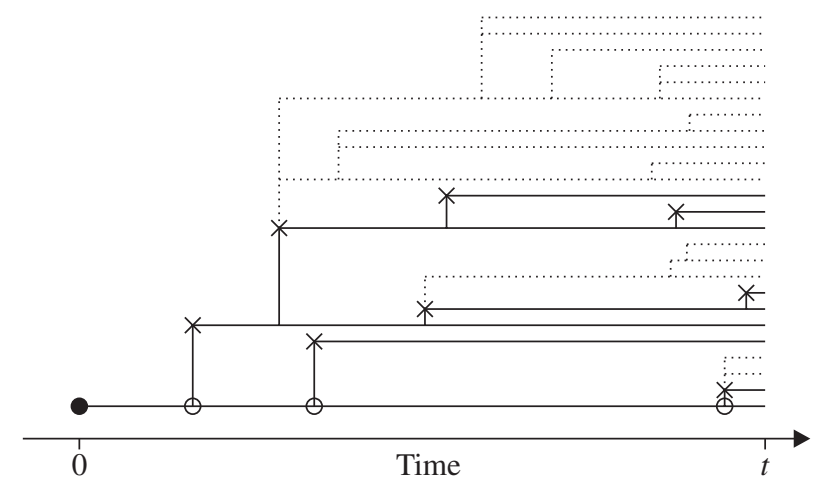

FIGURE 1: A realization of an immortal branching process. The open circles denote the times when the title is passed on under the scheme in Section 4; thus, they form a realization of a Poisson process. The crosses denote the times when the title is passed on under the system in Section 6; they have probability density $h(r)$. The lineages of individuals with the title under the second scheme are denoted by solid lines, and lineages without the title are denoted by dotted lines.

where $H(s, t)$ is a GF. Differentiating with respect to $t$ gives us

$$
\frac{\partial}{\partial t} F(s, t)=\mu F(s, t)\left(\frac{F(s, t)}{s}\left(H(s, t)+\frac{\mathrm{e}^{\mu t}-1}{\mu} \frac{\partial}{\partial t} H(s, t)\right)-1\right) .
$$

By comparing this with (5), we get

$$
H(s, t)+\frac{\mathrm{e}^{\mu t}-1}{\mu} \frac{\partial}{\partial t} H(s, t)=\operatorname{sk}(F(s, t)),
$$

which has the solution

$$
H(s, t)=\frac{\mu s}{1-\mathrm{e}^{-\mu t}} \int_{0}^{t} \mathrm{e}^{-\mu u} k(F(s, u)) \mathrm{d} u=s \int_{0}^{t} k(F(s, t-r)) h(r) \mathrm{d} r,
$$

which really is a GF since $k(F(s, t))$ is a GF $(h(r)$ is the probability density defined by $(9))$.

We can give a nice probabilistic interpretation of this result if we compare the general immortal branching processes with the simple Yule process. Imagine, as before, that the ancestor has a certain title that he passes on, but let us change the rules of inheritance so that two of the offspring inherit the title. Now we can consider the group of individuals that have the title at any given time $t$. This group will form a Yule process $\widehat{Z}$ with intensity $\mu$. Thus, $\widehat{Z}_{t}-1$ has a geometric distribution with parameter $1-\mathrm{e}^{-\mu t}$. The rest of the population originates from siblings of individuals in the Yule process $\widehat{Z}$. The number of siblings of two individuals who were given the title has GF $k(s)$, and each of those siblings will produce a subtree of its own with total size having GF $F(s, t-r)$, if that sibling is born at time $r$. Thus, the size of all subtrees of siblings of a given pair of title-bearers, born at time $r$, has GF $k(F(s, t-r))$. Finally, we know from Lemma 2 that the unconditional distribution of a time of birth in the Yule process $\widehat{Z}_{t}$ has distribution $h(r)$. See Figure 1 for an illustration.

Theorem 2 also provides information about the limiting random variable $W$ of Proposition 1.

Corollary 2. Let $Z$ be a supercritical branching process and $W$ be the limiting random variable of Proposition 1. Assume that (3) holds. Then $W$ has a compound geometric distribution and $W$, given that $W>0$, has a compound exponential distribution. 
Proof. If we repeat the proof of Corollary 1 with the information that $\widetilde{Z}_{t}$ is not only infinitely divisible but also compound geometric, we obtain, using Proposition 4 , the result that $W$, given that $W>0$, has a compound exponential distribution. By Lemma 1, this implies that $W$ has a compound geometric distribution.

\section{Another branching distribution}

So far, we have only seen quite trivial immortal branching processes with $p_{k}=1$ for some $k \geq 2$. There is another class of immortal branching processes whose marginal distribution can be found explicitly. Let

$$
p_{k}=\frac{(\gamma+1) \Gamma(k-1-\gamma)}{k ! \Gamma(1-\gamma)}=(\gamma+1) \frac{(1-\gamma)(2-\gamma) \cdots(k-2-\gamma)}{k !},
$$

for $0<\gamma<1$ and $k \geq 2$. For $\gamma=1$ we set $p_{2}=1$, and for $\gamma=0$ we set $p_{k}=1 /(k(k-1))$. The GF is

$$
f(s)= \begin{cases}(1-s) \frac{(1-s)^{\gamma}-1}{\gamma}+s & \text { for } 0<\gamma \leq 1, \\ (1-s) \log (1-s)+s & \text { for } \gamma=0 .\end{cases}
$$

We note that $\gamma=1$ is the ordinary Yule case, so this family of branching processes can be seen as a generalization of the Yule process.

In the case $\gamma=0$, the expected number of offspring at each birth is infinite, i.e. $m=f^{\prime}(1)=$ $\infty$, so Proposition 1 does not hold and there is no limiting random variable $W$. Nonetheless, the integral in (1) diverges, so the branching process does not explode in finite time almost surely. The solution of the Kolmogorov backward equation (4) when $\gamma=0$ is

$$
F(s, t)=1-(1-s)^{\exp (-\mu t)} .
$$

A branching process with offspring distribution $\left\{p_{k}\right\}$ with $0<\gamma<1$ has appeared in the theory of coalescent processes [2] and continuous (stable) random trees [4]. The solution of (4) is now, when $0<\gamma \leq 1$, given by

$$
F(s, t)=1-\frac{1}{\left(1-\mathrm{e}^{-\mu t}+\mathrm{e}^{-\mu t}(1-s)^{-\gamma}\right)^{1 / \gamma}} .
$$

We see that this result is in concordance with (12) for $\gamma=1$.

The expected number of offspring at each birth is $m=f^{\prime}(1)=1+1 / \gamma$. The limiting random variable $W$ of (2) has Laplace-Stieltjes transform

$$
\begin{aligned}
L_{W}(\theta) & =\lim _{t \rightarrow \infty} \mathrm{E}\left[\exp \left(-\theta \mathrm{e}^{-\mu t / \gamma} Z_{t}\right)\right] \\
& =\lim _{t \rightarrow \infty} F\left(\exp \left(-\theta \mathrm{e}^{-\mu t / \gamma}\right), t\right) \\
& =\lim _{r \rightarrow 0} F\left(\mathrm{e}^{-\theta r},-\frac{\gamma}{\mu} \log r\right) \\
& =\lim _{r \rightarrow 0}\left(1-\frac{1}{\left(1-r^{\gamma}+r^{\gamma}\left(1-\mathrm{e}^{-\theta r}\right)^{-\gamma}\right)^{1 / \gamma}}\right) \\
& =1-\frac{1}{\left(1+\theta^{-\gamma}\right)^{1 / \gamma}} .
\end{aligned}
$$


Let $F_{\gamma}(x)$ be the distribution function of $W$, and $f_{\gamma}(x)$ its probability density, with Laplace transform $L_{W}(\theta)$. Then $1-F_{\gamma}(x)$ has Laplace transform

$$
\begin{aligned}
\frac{1}{\theta}-\frac{1}{\theta} L_{W}(\theta) & =\frac{1}{\left(1+\theta^{\gamma}\right)^{1 / \gamma}} \\
& =\theta^{-1}\left(1+\theta^{-\gamma}\right)^{-1 / \gamma} \\
& =\sum_{k=0}^{\infty}\left(\begin{array}{c}
-1 / \gamma \\
k
\end{array}\right) \theta^{-\gamma k-1} .
\end{aligned}
$$

By inverting this series term by term we obtain

$$
\begin{aligned}
1-F_{\gamma}(x) & =\sum_{k=0}^{\infty}\left(\begin{array}{c}
-1 / \gamma \\
k
\end{array}\right) \frac{x^{\gamma k}}{\Gamma(1+\gamma k)} \\
& =\frac{1}{\Gamma(1 / \gamma)} \sum_{k=0}^{\infty} \frac{\Gamma(1 / \gamma+k)}{\Gamma(1+\gamma k)} \frac{\left(-x^{\gamma}\right)^{k}}{k !} .
\end{aligned}
$$

We note that $F_{1}(x)=1-\mathrm{e}^{-x}$, as expected. When $0<\gamma<1$, this series is a special case of several different special functions. With the notation of [6, Equation (1.7.8)],

$$
\begin{aligned}
1-F_{\gamma}(x) & =\frac{1}{\Gamma(1 / \gamma)} 1_{1} \Psi_{1}\left[\begin{array}{cc}
(1 / \gamma, 1) ;-x^{\gamma} \\
(1, \gamma) ;
\end{array}\right] \\
& =\frac{1}{\Gamma(1 / \gamma)} H_{1,2}^{1,1}\left[\begin{array}{c|cc}
x^{\gamma} \mid \begin{array}{cc}
(1-1 / \gamma, 1) \\
(0,1)
\end{array} & (0, \gamma)
\end{array}\right],
\end{aligned}
$$

where $\Psi$ is a certain generalization of the hypergeometric function and $H$ is the Fox $H$-function. For rational values of $\gamma, 1-F_{\gamma}(x)$ can be expressed with the Meijer $G$-function, which has the advantage of being implemented in software packages such as MATHEMATICA ${ }^{\circledR}$ and MAPLE $^{\circledR}$. For example, if $\gamma=p / q$ with $p$ and $q, p<q$, being two relatively prime positive integers, then, by [7, Equation (8.3.2.22)],

$$
\begin{aligned}
1-F_{p / q}(x)= & \frac{q^{q / p}}{\Gamma(q / p) \sqrt{p}(\sqrt{2 \pi})^{2 q-p-1}} \\
& \times G_{q, p+q}^{q, q}\left(\left(\frac{x}{p}\right)^{p} \mid \begin{array}{c}
1 / q-1 / p, 2 / q-1 / p, \ldots, 1-1 / p \\
0,1 / q, \ldots,(q-1) / q, 0,1 / p, \ldots,(p-1) / p
\end{array}\right) .
\end{aligned}
$$

Remark 2. The limit distribution (13) also appears for critical branching processes with infinite variance for the offspring distribution, conditioned on nonextinction; see [9].

\section{References}

[1] Athreya, K. B. And Ney, P. E. (1972). Branching Processes. Springer, New York.

[2] Berestycki, J., Berestycki, N. and Schweinsberg, J. (2006). Beta-coalescents and continuous stable random trees. To appear in Ann. Prob.

[3] BüHLER, W. J. (1968). Some results on the behaviour of branching processes. Theory Prob. Appl. 13, 52-64.

[4] Duquesne, T. and Le Gall, J.-F. (2002). Random trees, Lévy processes and spatial branching processes. Astérisque 281, 147pp.

[5] Harris, T. E. (1963). The Theory of Branching Processes. Springer, Berlin. 
[6] Mathai, A. M. and Saxena, R. K. (1978). The H-function with Applications in Statistics and Other Disciplines. John Wiley, New York.

[7] Prudnikov, A. P., Brychkov, J. A. and Marichev, O. I. (1990). Integrals and Series, Vol. 3, More Special Functions. Gordon and Breach, New York.

[8] Steutel, F. W. and van Harn, K. (2004). Infinite Divisibility of Probability Distributions on the Real Line. Marcel Dekker, New York.

[9] Zolotarev, V. M. (1957). More exact statements of several theorems in the theory of branching processes. Theory Prob. Appl. 2, 245-253. 